Wojciech Kosek, North Dakota State University, Fargo, ND 58105 e-mail:

kosek@@lanin.nodak.edu

\title{
ON "LIPSCHITZ" SUBSPACES OF THE SPACE OF CONTINUOUS FUNCTIONS
}

\begin{abstract}
A theorem of Grothendieck states that every closed subspace of the Banach space $L^{p}(\mu)$, where $\mu$ is a finite measure on a locally compact topological space, $p \geq 1$, consisting of essentially bounded functions must have finite dimension. An analog of this result is proved concerning subspaces of the space of continuous functions on a compact metric space consisting of functions satisfying different Lipschitz-type conditions.
\end{abstract}

\section{Introduction}

Consider a Banach space $X$ and a closed subspace $S$. In some cases we can say that every element of $S$ is "better" than an arbitrary element of $X$. This happens, for example, if elements of $X$ are functions defined on a metric space and elements of $S$ satisfy some additional estimates or smoothness conditions. A general question is how big such "good" subspaces can be.

A. Grothendieck [1] proved a very interesting result showing that it is natural to expect, at least in some cases, that these subspaces are small. Let $M$ be a locally compact topological space equipped with a finite measure $\mu$ and let $1 \leq p<\infty$. If $S$ is a vector subspace of $L^{\infty}(\mu)$, closed in $L^{p}(\mu)$, then $S$ is finite-dimensional.

\section{Preliminaries}

Let $K$ be a compact metric space and let $C(K)$ be a Banach space of all continuous complex valued functions on $K$. For any $f \in C(K)$ the modulus of continuity $\omega_{f}:(0, \infty) \rightarrow[0, \infty)$ is defined as

$$
\omega_{f}(\delta)=\sup _{\rho(x, y)<\delta}|f(x)-f(y)| .
$$

Clearly $\omega_{f}$ is an increasing function and $\lim _{\delta \rightarrow 0} \omega_{f}(\delta)=0$.

Key Words: Banach space, Closed Graph Theorem

Mathematical Reviews subject classification: Primary: 26A16 Secondary: 46E15

Received by the editors June 1, 1994 
Definition 1 Let $\omega:(0, \infty) \rightarrow(0, \infty)$. We will say that $\omega$ is modulus-type if

(i) $\lim _{\delta \rightarrow 0} \omega(\delta)=0$,

(ii) $\omega$ is increasing.

By $S$ we will denote a closed, linear subspace of $C(K)$; its dimension is denoted by $\operatorname{dim} S$. The following statement is a variation on a theme of Grothendieck.

Proposition 1 Let $\omega$ be a modulus-type function. If for every $f \in S$

$$
\sup _{\delta>0} \frac{\omega_{f}(\delta)}{\omega(\delta)}<\infty
$$

then $\operatorname{dim} S<\infty$.

\section{First Proof of Proposition 1}

A shorter proof of the Proposition using Ascoli's theorem and Riesz lemma will be outlined later. We would like to present a more direct approach. We start with the following simple observation.

Lemma 1 Suppose $\operatorname{dim} S=\infty$ and $\left\{x_{1}, x_{2}, \ldots, x_{n}\right\} \subset K$. Then there exists a function $f \in S$, such that $f \neq 0$, but $f\left(x_{1}\right)=f\left(x_{2}\right)=\cdots=f\left(x_{n}\right)=0$.

Proof. Suppose not. Consider a linear map $B: S \rightarrow \mathbb{R}^{n}$ given by

$$
B(f)=\left(f\left(x_{1}\right), f\left(x_{2}\right), \ldots, f\left(x_{n}\right)\right) .
$$

Clearly, B is linear and $\operatorname{ker} B=\{0\}$. Hence

$$
\operatorname{dim} S=\operatorname{dim}(\operatorname{ker} B)+\operatorname{dim}(B(S))=0+n=n .
$$

But this contradicts the assumption that $\operatorname{dim} S=\infty$.

Proof of the Proposition Let $\omega$ be a modulus type function. We shall show that there is a constant $M$ such that for all $\delta>0$

$$
\frac{\omega_{f}(\delta)}{\omega(\delta)} \leq M\|f\|_{C(K)}
$$

Let

$$
Y=\left\{f \in C(K): \sup _{\delta>0} \frac{\omega_{f}(\delta)}{\omega(\delta)}<\infty\right\} .
$$

If

$$
\|f\|_{Y}=\sup _{x \in K}|f(x)|+\sup _{\delta>0} \frac{\omega_{f}(\delta)}{\omega(\delta)}
$$


then $\left(Y,\|\cdot\|_{Y}\right)$ is a Banach space.

Consider an identity map $I: S \rightarrow Y$ i.e. $I f=f$ for $f \in S$. $(S$ is treated as a subspace of a Banach space $C(K)$.) Obviously $I$ is linear. It is easy to deduce from the Closed Graph Theorem that $I$ is also bounded as a linear operator. Let $\left\{f_{n}\right\}_{n \in \mathbb{N}}$ be a sequence of functions in $S$, such that $\left\|f_{n}-f\right\|_{S} \rightarrow 0$ and $\left\|I f_{n}-g\right\|_{Y} \rightarrow 0$. Then

$$
\left\|f_{n}-g\right\|_{C(K)} \leq\left\|f_{n}-g\right\|_{Y}=\left\|I f_{n}-g\right\|_{Y} .
$$

Hence $g=\lim _{n \rightarrow \infty} f_{n}$ and trivially $g=f$. Therefore by the Closed Graph Theorem $I$ is a bounded linear operator, i.e. there exists a constant $M_{1}$ such that for all $f \in S$ we have $\|f\|_{Y} \leq M_{1}\|f\|_{C(K)}$ Since $\|f\|_{Y}=\|f\|_{C(K)}+\sup _{\delta>0} \frac{\omega_{f}(\delta)}{\omega(\delta)}$, for $M=M_{1}-1$ we obtain (1).

Since $\lim _{\delta \rightarrow 0} \omega(\delta)=0$, let us fix $\delta_{0}>0$ such that $\omega\left(\delta_{0}\right)<\frac{1}{M} K$ is a compact metric space, so there exists a $\delta_{0}$-net, i.e. a set

$\left\{x_{1}, x_{2}, \ldots, x_{n}\right\} \subset K$ such that for every $x \in K$ there is an $i \in\{1,2, \ldots, n\}$ such that $\rho\left(x, x_{i}\right)<\delta_{0}$.

Suppose that $\operatorname{dim} S=\infty$. By Lemma 1 , there is a function $f \in S, f \neq 0$, such that $f\left(x_{1}\right)=f\left(x_{2}\right)=\cdots=f\left(x_{n}\right)=0$. Since $K$ is compact, $\|f\|_{C(K)}=\left|f\left(x_{0}\right)\right|$ for some $x_{0} \in K$. Therefore, by (3) we obtain for some $i \in\{1,2, \ldots, n\}$

$$
\begin{aligned}
\omega_{f}\left(\delta_{0}\right) & =\sup _{\rho(x, y)<\delta_{0}}|f(x)-f(y)| \geq\left|f\left(x_{0}\right)-f\left(x_{i}\right)\right| \\
& =\left|f\left(x_{0}\right)-0\right|=\|f\|_{C(K)}=M\|f\|_{C(K)} \frac{1}{M} \\
& >M\|f\|_{C(K)} \omega\left(\delta_{0}\right)
\end{aligned}
$$

or $\frac{\omega_{f}\left(\delta_{0}\right)}{\omega\left(\delta_{0}\right)}>M\|f\|_{C(K)}$ contrary to (1).

\section{Second Proof of Proposition 1}

We used the Closed Graph Theorem to show that norms $\|\cdot\|_{C(K)}$ and $\|\cdot\|_{Y}$ are equivalent on $S$. The same fact can be established if we apply Banach's theorem on isomorphism. Let us consider $\Phi=S \cap\left\{f \in C(K):\|f\|_{C(K)} \leq 1\right\}$. From the equivalence of norms $\|\cdot\|_{C(K)}$ and $\|\cdot\|_{Y}$ we obtain that $\Phi$ is an equicontinuous family of functions in $C(K)$, bounded by 1. Since $\Phi$ is closed, Ascoli's theorem gives compactness of $\Phi$. But the unit ball in a Banach space is compact if and only if its dimension is finite (the Riesz lemma). Hence $\operatorname{dim} S<\infty$.

\section{Consequences}

Let us notice some straightforward corollaries from the Proposition: 
Corollary 1 If $S \subset \operatorname{Lip}_{\alpha}(K)$, then $\operatorname{dim} S<\infty$.

Proof. Take $\omega(\delta)=\delta^{\alpha}$ and apply Proposition.

Corollary 2 If $S$ is a closed linear subspace of $C[0,1]$ and every $f \in S$ is continuously differentiable $\left(S \subset C^{1}[0,1]\right)$, then $\operatorname{dim} S<\infty$.

Proof. Since $C^{1}[0,1] \subset \operatorname{Lip}[0,1]$, this follows from Corollary 1.

The above corollary is one more statement illustrating the well known fact that a "typical" continuous function is not differentiable ([2]).

\section{Example}

It is required in Corollary 2 that all functions in $S$ have continuous derivatives on $[0,1]$. This example shows that it is not enough to assume only the existence of such derivatives on a subset of $[0,1]$.

It is well known that given an interval $(a, b)$, there exists a function $f_{a, b} \in$ $C^{\infty}(\mathbb{R})$ such that

(i) $f_{a, b}(x)=0$ for all $x \in \mathbb{R} \backslash(a, b)$,

(ii) $\sup _{x \in \mathbb{R}}\left|f_{a, b}(x)\right|=1$.

We can take for instance

$$
f_{a, b}(x)= \begin{cases}c_{a, b} e^{-\frac{1}{(x-a)^{2}(x-b)^{2}}}, & x \in(a, b) \\ 0, & x \in \mathbb{R} \backslash(a, b)\end{cases}
$$

where $c_{a, b}=e^{\frac{16}{(a-b)^{4}}}$. Let $g_{n}=f_{2^{-n}, 2^{-n+1}}$ and let $S$ be the closed linear manifold generated by $\left\{g_{n}\right\}_{n=1}^{\infty}$. Then $\operatorname{dim} S=\infty$ and every function in $S$ has continuous derivatives in $(0,1]$. Also $g=\sum_{n=1}^{\infty} \sqrt{n} g_{n} \in S$ and $g$ has no derivative at 0 .

\section{Acknowledgment}

The author would like to express his gratitude to Professor Isaak Kornfeld for his comments and encouragement to write this paper.

\section{References}

[1] A. Grothendieck, Sur certains sous-espaces vectoriels de $L^{p}$, Can. J. Math., 6 (1954), 157-160.

[2] T. Pytlik and J. Gorniak, Functional Analysis in Exercises, Technical University of Wroclaw, Wroclaw, 1992, 84. 